\title{
ALTERNATIVA CURRICULAR PARA LA ENSEÑANZA DEL FÚTBOL BASE \\ "Una experiencia de investigación en las escuelas deportivas de la ciudad de Neiva"
}

Virgilio Puerto Polanco ${ }^{44}$

\section{RESUMEN}

El artículo "Alternativa Curricular para la Enseñanza del Fútbol-Base" que se presenta en las líneas posteriores de este texto se constituye en el informe final de investigación realizado en el programa "Maestría en Educación con Énfasis en Diseño, Gestión y Evaluación Curricular" de la Universidad Surcolombiana.

En él se dan a conocer los resultados del estudio realizado entre los años 2009 y 2010 sobre la identificación y establecimiento de una Programación Curricular para la Enseñanza del Fútbol Base en la ciudad de Neiva con niños cuyas edades están entre los 8-10 años. Parte de la información que se entrega está relacionada con referentes teóricos que definen y caracterizan el tema del currículo como una alternativa de vital importancia para la organización y dinámica de funcionamiento de los procesos de formación deportiva que giran alrededor del fútbol-base.

El referente teórico construido en este trabajo de investigación también hace referencia a conceptos y particularidades que tienen que ver con el tratamiento pedagógico y didáctico del fútbol, tendencias actuales sobre el fútbol base, y enfoques metodológicos de mayor utilización.

De la misma manera, se presenta en el aparte denominado "resultados de investigación" un avance importante en la identificación y constitución de contenidos, temas y metodologías para orientar la práctica del fútbol base con mayor sentido y apropiación en los niños, ya que se definen "Las Unidades de Práctica". Dichas unidades se caracterizan por la inclusión de diversos temas, que ha manera de juego hacen de la sesión de entrenamiento, un espacio de recreación, expresión lúdica y desarrollo de la personalidad.

PALABRAS CLAVES: Currículo, fútbol base, unidades de práctica, pedagogía y didáctica del fútbol. 


\section{OBJETIVOS}

\section{Objetivo Generall}

- Definir cuál es la estructura curricular que requieren las escuelas de formación deportiva del municipio de Neiva para la enseñanza del fútbol-base con niños de 8 a 10 años.

\section{Objetivos Específicos}

- Establecer cómo son las estructuras curriculares de las escuelas de formación deportiva para la enseñanza del fútbol-base en el municipio de Neiva.

- Identificar mancomunadamente con los entrenadores del fútbol-base del municipio de Neiva aspectos de tipo metodológico, pedagógico y didáctico encauzados a elaborar una alternativa curricular para las escuelas de formación deportiva.

- Identificar elementos fundamentales del "juego" para establecer a partir de éste, estrategias pedagógicas y didácticas en el proceso formativo del fútbol-base.

\section{METODOLOGÍA}

\section{Tipo de lnvestigación.}

Esta investigación se desarrolló teniendo en cuenta un enfoque metodológico de tipo cualitativo, en donde se acudió de manera fundamental a la Investigación Participación (IP). Torres Carrillo (1995-86) citando a Cendales considera que "esta es una propuesta metodológica que forma parte de una estrategia que involucra a la comunidad en el conocimiento y solución de sus problemas; centra su objeto de conocimiento y finalidad en la práctica; parten de ella y vuelven a ella para cambiarla" además, su dinámica de desarrollo "implica la participación activa y decisoria de los sujetos involucrados. Investigadores e investigados son al mismo tiempo objeto y sujeto de la investigación".

Desde esta perspectiva la investigación "Alternativa Curricular para la Enseñanza del Fútbol-Base" hizo énfasis en:

- Involucrar de manera activa en la investigación a los entrenadores, padres de familia y niños de las escuelas deportivas seleccionadas para el estudio.

- Centrar como "objeto de conocimiento y finalidad" de la investigación las 
prácticas deportivas del fútbol que realizan los niños para analizar e interpretar sus dinámicas de funcionamiento y posteriormente proponer nuevas alternativas de trabajo, es decir, "transformar las prácticas"

- Vincular a los entrenadores como coinvestigadores (sujeto investigador) y a partir de sus vivencias y experiencia en el contexto de la práctica del fútbol base (objeto de investigación) construir una propuesta para la realización de las sesiones de entrenamiento con los niños.

\section{UNIDAD DE ANÁLISIS Y UNIDAD DE TRABAJO.}

\section{Unidad de Análisis.}

La unidad de análisis para el desarrollo de esta investigación estuvó compuesta por todas las escuelas de formación deportiva existentes en la ciudad de Neiva que cuenten con procesos de formación del Fútbol-Base con niños de 8 a 10 años. La cantidad de escuelas existentes en el municipio de Neiva es de 23 y la relación de las mismas es la siguiente:

\begin{tabular}{|c|l|l|l|}
\hline No & NOMBRE DE LA ESCUELA & REPRESENTANTE LEGAL & \multicolumn{1}{|c|}{ TELEFONO } \\
\hline 1 & SURNEY & EVER BURGOS & 8716949 \\
\hline 2 & VILLAMILENA J.R. & JAIME RODRIGUEZ & 3105576618 \\
\hline 3 & ROBERTO CARLOS & ROBERTO ATEHORTUA & 8746062 \\
\hline 4 & LUDENS & JOSE REYNALDO CHICA & 8715355 \\
\hline 5 & OLAYA HERRERA & FREDY PARDO & 8756464 \\
\hline 6 & COMFAMILIAR HUILA & ARMANDO ARIZA & 8764321 \\
\hline 7 & CHICOS FUTBOL CLUB & FERNANDO MOTTA & 8734488 \\
\hline 8 & E.H.C. & EDUARDO HERNANDEZ & 3152984492 \\
\hline 9 & SPORTING 99 & BENJAMIN GONZALEZ & 3002585450 \\
\hline 10 & UTRAHUILCA & JOSE HOBER PARRA & 8754508 \\
\hline 11 & CHOCHOS & ALVARO CARDOZO & 3162386829 \\
\hline 12 & SOCIAL Y DEPORTIVO GRANJAS & JORGE MOLINA & 8753552 \\
\hline 13 & TIGRES DEL SUR & JORGE MORALES & 3174283208 \\
\hline 14 & HUILA SIGLO XXI & RAFAEL CARDENAS & 8671300 EXT 1112 \\
\hline 15 & CYCLONES & JAIRO SILVA & 3132513506 \\
\hline 16 & TEACHER BERRIO & LUIS GUILLERMO BERRIO & 3158748188 \\
\hline
\end{tabular}




\begin{tabular}{|c|l|l|l|}
\hline 17 & $\begin{array}{l}\text { CORPORACION DEPORTIVA } \\
\text { DEL HUILA }\end{array}$ & YEISON NARANJO & 8756732 \\
\hline 18 & DEPORNEIVA & JESUS BUSTOS & 3203269369 \\
\hline 19 & AMIGOS COMO ARROZ & YILBER CEJAS & 8708670 \\
\hline 20 & USCO & DIEGO QUIMBAYA & 3112614589 \\
\hline 21 & U.C.C. & JORGE MUÑOZ & 3158985382 \\
\hline 22 & ORIENTE & DIEGO GRANADOS & 3213043117 \\
\hline 23 & MARTIRES & ANA DE JESUS ORTIZ & 3172701926 \\
\hline
\end{tabular}

\section{Unidad de Trabajo.}

La unidad de trabajo para esta investigación la constituyeron 10 escuelas de formación deportiva en fútbol. La siguiente es la relación de las escuelas seleccionadas para el estudio:

\begin{tabular}{|c|l|l|l|}
\hline No & \multicolumn{1}{|c|}{ NOMBRE DE LA ESCUELA } & REPRESENTANTE LEGAL & \multicolumn{1}{|c|}{ TELEFONO } \\
\hline 1 & VILLAMILENA J.R. & JAIME RODRIGUEZ & 3105576618 \\
\hline 2 & LUDENS & JOSE REYNALDO CHICA & 8715355 \\
\hline 3 & E.H.C. & EDUARDO HERNANDEZ & 3152984492 \\
\hline 4 & UTRAHUILCA & JOSE HOBER PARRA & 8754508 \\
\hline 5 & CHOCHOS & ALVARO CARDOZO & 3162386829 \\
\hline 6 & HUILA SIGLO XXI & RAFAEL CARDENAS & 8671300 EXT 1112 \\
\hline 7 & TIGRES DEL SUR & JORGE MORALES & 3174283208 \\
\hline 8 & TEACHER BERRIO & LUIS GUILLERMO BERRIO & 3158748188 \\
\hline 9 & DEPORNEIVA & JESUS BUSTOS & 3203269369 \\
\hline 10 & MARTIRES & ANA DE JESUS ORTIZ & 3213043117 \\
\hline
\end{tabular}

Los criterios que se utilizaron para la selección de la unidad de trabajo fueron los siguientes:

- Que los entrenadores del proceso de formáción mostraran interés y se comprometieran con la participación activa en el proyecto desde el inicio y hasta el final del mismo.

- Que la escuela deportiva cuente con niños cuyas edades estén entre los 8-10 años dado que este es el rango de edad definido para trabajar con la población seleccionada. 
- Que las escuelas deportivas cuenten con la infraestructura y el material didáctico adecuado para el desarrollo de las prácticas.

- Que las escuelas deportivas se encuentren ubicadas en la zona urbana de la ciudad de Neiva y que geográficamente estén funcionando en diferentes comunas.

\section{Técnicas e Innstrumentos utillizados para Recoger la Información.}

Relacionado con este aspecto, para el desarrollo de la investigación se utilizó el taller y la ficha de observación. Cada uno de los anteriores se describe a continuación:

Ell Taller: Los talleres consistieron en el desarrollo de actividades con los entrenadores de las escuelas de formación donde se presentarán diferentes temáticas relacionadas con el tema objeto de esta investigación. La dinámica de los talleres estuvó constituida por diferentes sesiones a saber: temas, objetivos, compromisos, espacios de discusión, entre otros. En el anexo número uno se presenta un ejemplo de la estructura del taller

La Ficha de Observación: La ficha de observación se utilizó con el objetivo de obtener información sobre el diagnostico hecho a las escuelas deportivas y sobre la aplicación de las Unidades de Práctica. Bonilla y Rodríguez (2000) precisan que "observar implica focalizar la atención de manera intencional sobre algunos segmentos de la realidad que se estudia, tratando de captar sus elementos constitutivos y la manera cómo interactúan entre sí, con el fin de reconstruir inductivamente la dinámica de la situación". Esta fué aplicada por los entrenadores de las escuelas y periódicamente el autor de este estudio realizó visitas para corroborar que se aplicaran con la mayor objetividad posible.

\section{Procedimiento Utillizado para Recoger la Información.}

El procedimiento para llevar a cabo la recolección de los datos estuvó constituido por tres etapas a saber:

La primera consistió en el desarrollo de un diagnostico que permitió establecer cómo estaban constituidas las escuelas de formación deportiva de fútbol en cuanto a su organización, estructura y programación curricular, estado de conservación de los escenarios deportivos donde se llevan a cabo las prácticas y otros aspectos importantes que se deriven de este estudio. En el anexo número dos se presenta el instrumento guía que se utilizó en esta etapa. 
La segunda etapa estuvó determinada por la elaboración y aplicación de las Unidades de Práctica defínidas por el equipo de investigación (investigador principal, auxiliares de investigación y entrenadores). Estas se utilizaron como los contenidos temáticos que orientaron con los niños en las escuelas de formación deportiva durante el año 2010.

La tercera etapa correspondió a los talleres para integrar los productos, resultado del análisis y discusión con los entrenadores deportivos, quienes a partir de sus vivencias aportaron en la construcción de la Alternativa Curricular para la Enseñanza del Fútbol Base, producto final de esta investigación.

\section{SOBRE LA ALTERNATIVA CURRICULAR PARA LA ENSEÑANZA DEL FÚTBOL- BASE。}

Dada las breves particularidades anotadas anteriormente sobre el concepto, la interpretación y la evolución histórica y cultural del currículo, se precisa que la Alternativa Curricular para la Enseñanza del Fútbol-Base se constituye en parte, como la organización de los procesos para enseñar a los niños practicantes de este deporte, definiendo no solo los aprendizajes específicos del fútbol, sino apuntando a la construcción cultural, social y personal del infante. Se puede estimar también, como aquella posibilidad que tiene el docente de contar con referentes teóricos que le permitan orientar su proceso de enseñanza-aprendizaje de manera metódica, organizada y consecuente con la implementación de temas que se realicen desde diferentes ámbitos del desarrollo humano.

Esta propuesta curricular posee su fundamento en los postulados que llevan a inferir que la selección y organización de los temas a enseñar deben ser estructurados, de manera fundamental, a partir de los siguientes aspectos: el contexto, las características particulares de los beneficiarios (los niños) y el proceso de investigación.

Se debe entender el contexto en un sentido amplio, teniendo en cuenta que no es solamente el escenario donde se desarrolla el proceso deportivo, sino diferentes aspectos que lo constituyen, entre ellos, el sentir de la comunidad, los factores sociales, políticos, culturales, religiosos y académicos.

En cuanto a las características particulares de los beneficiarios, en este caso se habla de los niños (como beneficiarios directos) se debe tener en cuenta el perfil de desarrollo de los mismos desde el punto de vista físico, psicológico y motivacional. Desde estos aspectos el niño posee las condiciones ideales para iniciar un proceso de formación en el fútbol-base. 
Sobre el proceso de investigación se considera que es un factor fundamental para la elaboración de la Alternativa Curricular dado que se parte de una actividad de indagación y descubrimiento de factores que se asocian directamente con las necesidades reales que presentan los actores del estudio.

Desde esta perspectiva se acude a la organización de los temas a enseñar teniendo en cuenta varios elementos, entre ellos, las necesidades de aprendizaje de los niños, la tendencia metodológica utilizada por el profesor, la intensidad horaria, el perfil del entrenador y de manera especial la constitución de las sesiones de entrenamiento a través de Unidades de Práctica.

\section{PERSPECTIVA PEDAGÓGICA.Y DIDÁCTICA DEL FÚTBOL BASE.}

La lenta evolución de la pedagogía y la didáctica en los procesos deportivos, si se compara con el tratamiento y renovación pedagógica y didáctica de otros campos, ubica al deporte en un gran retraso. Dicha situación supone la necesidad de encontrar un camino que permita la utilización de estos conceptos en la práctica del fútbol.

Se considera entonces, que el éxito de los procesos de enseñanza aprendizaje se da, en gran medida, con la utilización de enfoques pedagógicos y didácticos adecuados a las distintas características que poseen los actores del proceso formativo. La pedagogía, para el caso de la enseñanza del fútbol-base debe considerarse como una interacción entre formador y deportista que tiene como objeto final del primero, la responsabilidad de liderar el proceso de formación deportiva sobre el segundo; conceptualizando y aplicando los conocimientos específicos del fútbol y a la vez, creando un ser humano con un carácter crítico, reflexivo y creativo. Por su lado la didáctica debe entenderse como las acciones utilizadas para enseñar y aprender de manera organizada un tema o una situación determinada que se presenta alrededor del fútbol.

Blásquez (1995-35) precisa que "el momento en que el niño empieza el aprendizaje en forma específica de los deportes, se puede identificar con el término de "iniciación deportiva". Ésta situación, sencilla y desprevenida a primera vista, resulta interesante y motiva un análisis a profundidad, sobre todo si se enfoca desde una perspectiva pedagógica".

En este sentido la iniciación deportiva plantea la necesidad de proponer un ambiente didáctico que defina explícitamente las aspiraciones e intereses del niño y el proceder del formador. En cuanto al niño se debe dar un espacio para la espontaneidad, la improvisación y la libertad física y lúdica, otorgándole a éste el valor que merece en el proceso deportivo. 
Dentro del ámbito pedagógico el formador debe caracterizarse por asumir la responsabilidad de hacer una profunda reflexión en cuanto a su quehacer diario en la formación de los niños futbolistas y en relación al método que más se ajusta a las posibilidades físicas de los mismos. Uno de los métodos más importantes para el proceso de enseñanza-aprendizaje es el activo (global), puesto que toma el juego como un factor motivador y canalizador de aprendizajes perceptivo-motrices y de habilidades que el niño ha ido adquiriendo en su proceso de desarrollo.

Ruiz, García y Casimiro (2001) afirman que "la utilización del juego, especialmente del juego colectivo, siendo éste algo intrínseco al niño, debe ser aprovechado para la adquisición de las destrezas, habilidades y técnicas de los distintos deportes colectivos, dejando para etapas posteriores la decantación por una especialidad concreta en función de sus posibilidades, cualidades físicas o simplemente, de sus preferencias".

Respecto a la didáctica empleada en la enseñanza del fútbol, Seybold (1965) asegura que "el éxito de la educación depende no solamente del método a utilizar, sino de la manera como se emplean estos métodos. El aprendizaje de cualquier modalidad deportiva debe ser hecho con una cuidadosa y adecuada metodología y debe estar en concordancia con el ideal educativo y la realidad del educando".

Bruggeman y Albrecht (1996) afirman que "se debe considerar que el solo practicar fundamentos técnicos sin la oportunidad de adquirir "experiencia de juego" con situaciones de competición que contengan aspectos técnicos y tácticos básicos con el stress del enfrentamiento, no derivará en el éxito de un proceso serio orientado al desarrollo integral del deportista".

\section{TENDENCIAS ACTUALES DEL FÚTBOL BASE.}

Existen varias concepciones que caracterizan el desarrollo de los procesos deportivos, dentro de las cuales se pueden destacar la concepción deportiva tradicional que generalmente busca conservar valores relacionados con el culto al rendimiento, la ley del más fuerte, el orden y el principio de autoridad y por otro lado, la concepción humanista caracterizada por el desarrollo de un potencial personal, objetivos a largo plazo, reivindicación de la libertad y un clima pedagógico apropiado.

Desde esta perspectiva, los objetivos en el Fútbol Base se pueden plantear desde dos ópticas diferentes, éstas se relacionan a continuación: 


Concepción Deportiva
Se expone al niño prematuramente a la
competición de los adultos. Dura años
hasta que desarrolle los mismos hábitos
que demuestran los adultos en el juego
11 contra 11.

El responsable es un entrenador/técnico que instruye con el fin de ganar el partido cer al técnico que manda o instruye desde la línea lateral.

En busca de la victoria se enseña a ser desleal, hacer trampas, ser deshonesto y engañar a los contrarios y al árbitro. Los fines justifican los medios.

Para poder ganar prevalece en los entrenamientos la aplicación de la metodología tradicional.

Se valora más lo deportivo que la persona. Se aceptan frecuentemente comportamientos discutibles para conseguir un buen resultado.

Se suele seleccionar a jugadores que están acelerados en sus capacidades físicas, especialmente a los más fuertes. Ellos son los titulares

Desde los ocho años se da una desmesurada importancia a la táctica colectiva. y el campeonato. El jugador debe obede-

\section{Concepción Humanista}

Se adapta a la competición en cada fase de la evolución del niño a sus capacidades físicas e intelectuales.

El responsable es un formador que estimula con el fin de mejorar el rendimiento del jugador y de su equipo. El jugador reflexiona en todo momento sin la necesidad de hacer movimientos automatizados.

Se enseña a la deportividad, a la honestidad, a respetar el reglamento y ser leal en la confrontación con los adversarios.

Para poder formar mejor al jugador debe prevalecer en las prácticas la aplicación de la metodología activa.

Se da prioridad a la formación de la persona por medio del deporte. Se utiliza el deporte como una escuela de la vida.

fuertes. Se prefiere jugadores con perspectivas en el manejo del balón e inteligencia. El buen comportamiento fuera y dentro del campo es uno de los criterios para la selección así como la voluntad de esforzarse.

El partido sirve para evaluar el grado de habilidad y ganar experiencia en la táctica.

Se aplica siempre el mismo sistema de juego.

Se varia frecuentemente el sistema de juego

Se busca inmediatamente la profundidad en el juego de ataque. El equipo tiene prisa y juega más rápido de lo que es capaz.

Pocas intensiones para construir el juego. Frecuentemente al balón no pasa por el medio campo y va directamente a los atacantes, mediante pases largos.

Se juega más contra que con el balón. No hay tiempo y espacio para amagues.
Prevalece la cultura de mantener la posesión del balón. No hay prisa en el juego.

El balón avanza generalmente de la defensa a los mediocampistas y de ellos a los atacantes, basándose el juego en los principios de la comunicación y cooperación.

Se juega con el balón, acariciándolo. Hay tiempo para ejecutar fintas y utilizar su fantasía e imaginación. 


\section{RESULTADOS DE INVESTIGACIÓN}

\section{Definición y constitución de la unidad de práctica. (conceptualización)}

El entrenamiento tanto del joven como del adulto debe obedecer a una planificación partiendo desde lo macro hasta lo micro, es decir, a partir de un plan general que constituya el desarrollo de varios niveles de formación hasta la elaboración del plan de práctica diario. Esta organización permite de manera específica la definición de los ejes temáticos que componen las sesiones de entrenamiento. Los contenidos y los ambientes que hacen parte del proceso de formación se convierten en elementos significativos para la enseñanza-aprendizaje en el Fútbol-Base; convirtiéndose además esto, en un aspecto que debe ser tratado con especial atención.

Por esta razón, se propone para la formación del niño en el Fútbol-Base la estructura de la Unidad de Práctica. Ésta se puede entender como la totalidad de tareas que debe realizar el niño en cada una de las sesiones. La Unidad de Práctica se considera como un acto pedagógico determinante en la enseñanzaaprendizaje de esta especialidad deportiva, que responde a las necesidades psicológicas, físicas, sociales y afectivas del niño practicante.

El propósito fundamental en este aparte es conceptualizar y presentar las Unidades de Práctica haciendo énfasis sea los juegos de iniciación, los juegos de habilidades y capacidades básicas, los juegos simplificados, los juegos de coordinación y el partido. Estos cinco elementos configuran la estructura central de la sesiones de entrenamiento.

Los anteriores elementos deben estar organizados de tal manera, que en la práctica se observe un alto grado de motivación en los niños. Sobre este aspecto Martens, Christina, Harvey y Sharkey (1995) proponen que "para mantener los niveles de motivación se deben tener en cuenta los siguientes elementos: el nivel de dificultad de las tareas propuestas que debe ser acorde al nivel de habilidad del niño, las prácticas deben ser estimulantes y con amplia variedad de actividades, en todo momento se debe mantener en plena actividad a los niños y finalmente abstenerse de instruir y evaluar constantemente, ya que esto interrumpe la concentración en el desarrollo de las tareas" ${ }^{\prime \prime}$ Es importante anotar también, que la Unidad de Práctica tiene en cuenta los principios pedagógicos aplicados a la Educación Física que Ruiz Juan y Otros (2004) citando a Seybold resumen de la siguiente manera. 
Ilustración 5: Elementos que estructuran la Unidad de Práctica
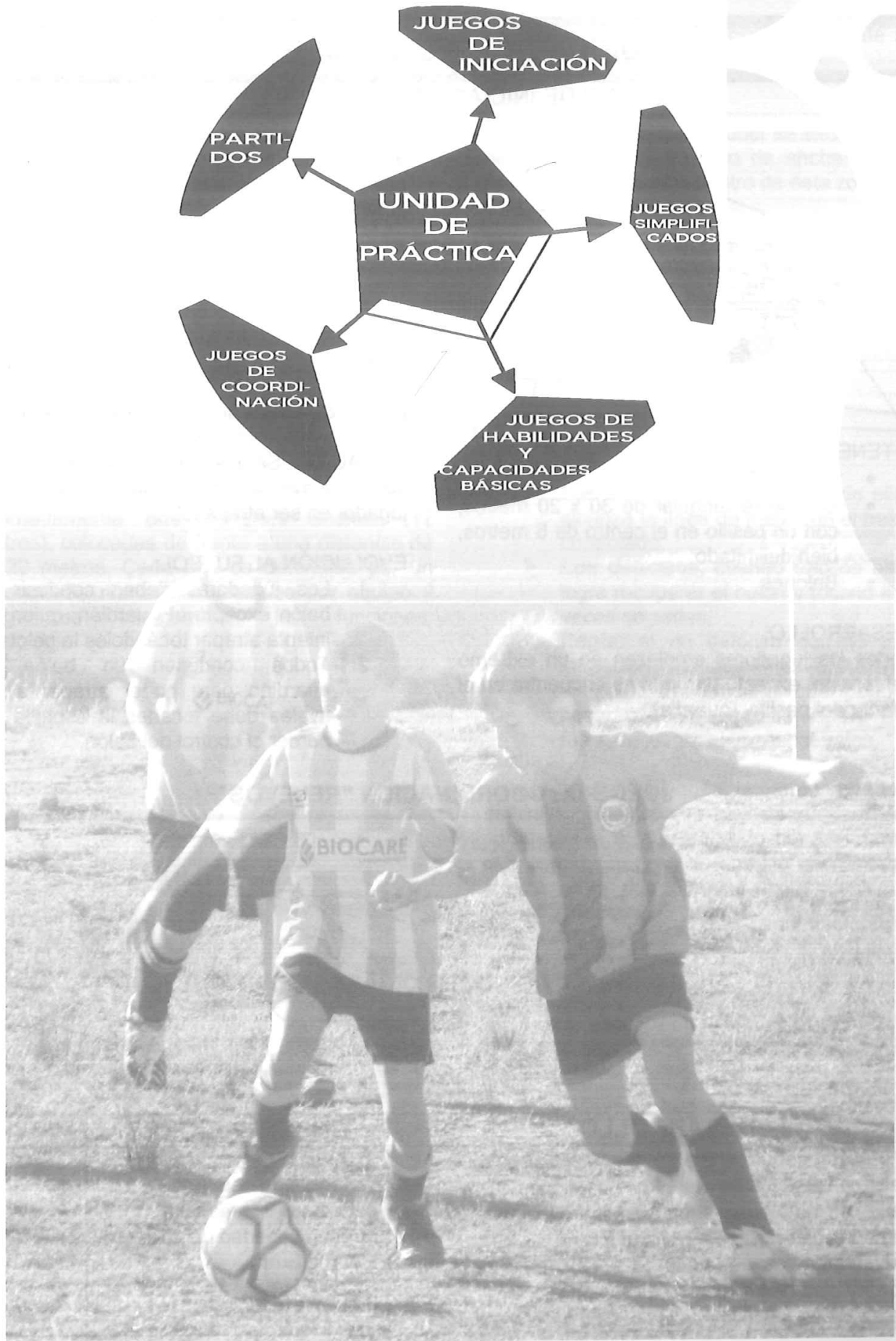
Presentación de las Unidades de Práctica. (la sesión)

\section{UNIDAD DE PRÁCTICA N ${ }^{\circ} 1$}

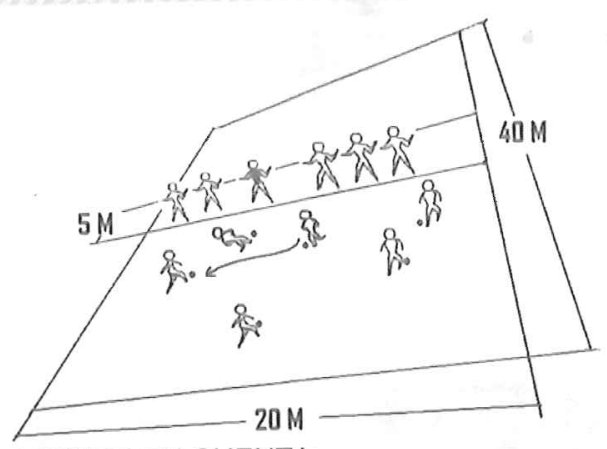

A TENER EN CUENTA:

- De 10 a 30 jugadores

- Campo rectangular de $30 \times 20$ metros, con un pasillo en el centro de 5 metros, bien delimitado.

- Balones

\section{DESARROLLO:}

Todos los jugadores empiezan en un extremo del terreno, excepto uno que se encuentra en el interior del pasillo (guardia).
A la señal todos los jugadores intentan pasar al otro extremo del terreno atravesando el pasillo. El guardián debe atrapar a los jugadores sin salirse del mismo.

Los jugadores atrapados son situados en el pasillo en el lugar exacto que ordene el guardián, permanecen inmóviles formando una muralla continua o varias pequeñas. De ésta manera disminuye el espacio para atravesar. Se elimina el jugador que tarde en pasar. Se puede autorizar atravesar el muro.

DURACIÓN: Se acaba el juego cuando todos se han convertido en muralla. Gana el último jugador en ser atrapado.

\section{EVOLUCIÓN AL FÚTBOL:}

1. Los jugadores deben conducir un balón excepto el guardián, quien los intenta atrapar tocándoles la pelota.

2. Todos conducen un balón, el guardián para poder atrapar a los rivales debe tocarles la espalda sin perder el control del balón.

\section{JUEGO DE COORDINACIÓN "RELEVOS"}

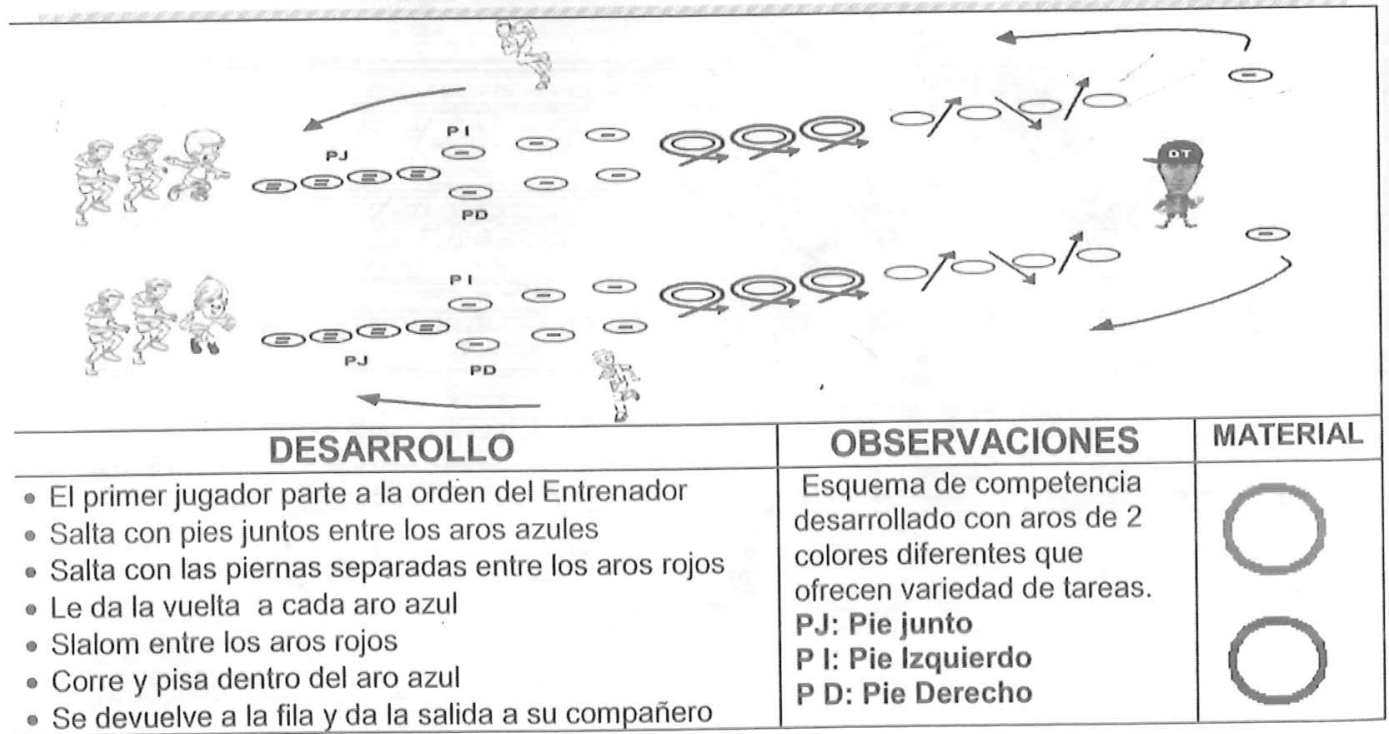


Un jugador desplaza el balón con un toque de cualquier pie a través de las piernas separadas del contrario, a la vez cuenta en voz alta los goles conseguidos durante 30 segundos. Se

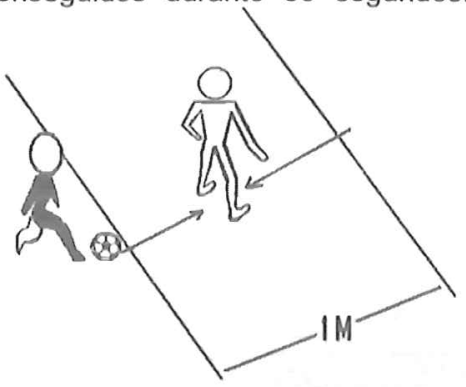

compite dos veces y en caso de empate se repite la prueba.

\section{VARIANTE 1:}

El jugador que forma el túnel se sitúa dentro de una franja de 1 metro de ancha, no se permite jugar el balón dentro de esta zona.

VARIANTE 2:

El jugador que forma el túnel, después de cada gol debe desplazar la posición del pie izquierdo (pívot) sin modificar la del pie derecho.

\section{JUEGO SIMPLIFICADO "2 CONTRA 1 A DOS PORTERIAS ANCHAS"}

Los dos jugadores de un equipo atacan alternadamente dos porterias anchas (12 metros), colocadas de frente a una distancia de 15-20 metros. Cada una es defendida por un jugador. Se realizan 10 ataques por equipo y luego se intercambian las funciones.

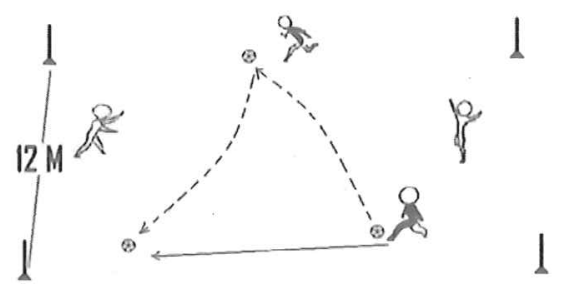

$15 \mathrm{M}$

El ataque termina con un punto a favor de:
Los atacantes, cuando uno de ellos alcanza la línea de meta con el balón controlado.

$>$ Los defensas, cuando uno de ellos logra recuperar el balón y tocarlo tres veces seguidas,

Regla: si un defensa comete una infracción, los atacantes ef́ectuarán un golpe franco en el cual pueden pasar o conducir el balón. No hay fuera de juego, ni líneas laterales.

Luego de los 10 ataques, la pareja ganadora se enfrenta a otra que haya triunfado en su competencia e igualmente lo hace la pareja que perdió. En caso de empate cada equipo realiza otros dos ataques hasta definir la pareja ganadora.

\section{PARTIDO "ESPACIO REDUCIDO 20 X 40 MTS"}

Juega el grupo A contra el grupo B. Cada equipo defiende y ataca las cuatro porterías. El número de jugadores por equipo: a partir de 4 contra 4.

\section{OBJETIVOS FORMATIVOS:}

- cambio de juego.

- Visión periférica. (campo visual)

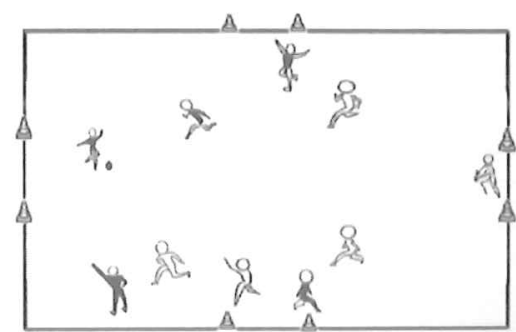


Tabla No 3. Principios Pedagógicos Aplicados a la Educación Física.

La inclinación del niño por el juego (situaciones reales globales).

La participación activa de los niños que favorezcan su libertad para crear movimientos.

El respeto al ritmo de ejecución y aprendizaje, por cuanto el niño debe expresarse conforme a su personalidad.

La adquisición de conciencia del niño, para encontrar su sitio en un colectivo.

La práctica debe ocupar un espacio en la formación integral del niño, en el desarrollo de su personalidad.

Todas las situaciones de enseñanza-aprendizaje deben ser significativas para el niño.

El niño asumirá rápidamente sus funciones de forma autónoma y la toma de decisiones, en cuanto a las tareas a desarrollar. 